Research Article

\title{
Retroesophageal Right Subclavian Artery
}

\author{
Neelesh Kanaskar, P. Vatsalaswamy, Preeti Sonje, and Vaishali Paranjape \\ Department of Anatomy, Dr. D. Y. Patil Medical College, Pimpri, Pune 18, India
}

Correspondence should be addressed to Neelesh Kanaskar; neeleshkanaskar@gmail.com

Received 12 July 2014; Accepted 16 September 2014; Published 2 October 2014

Academic Editor: Li-Hsien Lin

Copyright (C) 2014 Neelesh Kanaskar et al. This is an open access article distributed under the Creative Commons Attribution License, which permits unrestricted use, distribution, and reproduction in any medium, provided the original work is properly cited.

\begin{abstract}
During routine dissection of head and neck region in a 65-year-old female cadaver variation in the origin of right subclavian artery was found. The right subclavian artery originated as a direct branch of arch of aorta distal to the origin of left subclavian artery and it was found passing behind esophagus (retroesophageal) and ascending upwards to the right side while the left subclavian artery originated normally from arch of aorta distal to the origin of left common carotid artery. Anomalous variations in the origin and course of arteries have serious implications in angiographic and surgical procedures; hence it is of great importance to be aware of such possibilities of variations.
\end{abstract}

\section{Introduction}

Subclavian artery is the artery of the upper limb, but it also supplies a considerable part of the thoracic wall, neck, and brain through its branches.

On the left side it arises from arch of aorta and ascends on the pleura to enter the neck behind the left sternoclavicular joint. On the right side, it arises from the brachiocephalic artery behind the sternoclavicular joint. On each side it arches laterally across the anterior surface of cervical pleura onto the first rib, posterior to scalenus anterior. It becomes axillary artery at the outer border of first rib. The artery is divided into three parts by scalenus anterior muscle, and the second part of artery lies posterior to scalenus anterior [1].

Right subclavian artery may arise above or below sternoclavicular level. It may be a separate branch from aortic or the first or the last branch of the arch. Sometimes when the right subclavian artery is the last aortic branch, it passes between trachea and oesophagus and can cause dysphagia, a condition known as dysphagia lusoria. It may perforate scalenus anterior and very rarely may pass anterior to it. Sometimes the subclavian vein accompanies the artery behind scalenus anterior. The left subclavian artery is occasionally combined at its origin with the left common carotid artery [2].

\section{Case Report}

During routine dissection of head and neck region in a 65 -year-old female cadaver variation in the origin of right subclavian artery was found. The right subclavian artery originated as a direct branch of arch of aorta. It was seen arising as a last branch of arch of aorta distal to the origin of left subclavian artery and was found passing behind esophagus and ascending upwards to the right side while the left subclavian artery originated normally from arch of aorta distal to the origin of left common carotid artery (Figure 1).

\section{Discussion}

Classically subclavian artery on the left side arises from arch of aorta and ascends on the pleura to enter the neck behind the left sternoclavicular joint. On the right side, it arises from the brachiocephalic artery behind the sternoclavicular joint. On each side it arches laterally across the anterior surface of cervical pleura onto the first rib, posterior to scalenus anterior. It becomes axillary artery at the outer border of first rib. The artery is divided into three parts, and the second part of which lies posterior to scalenus anterior [1].

Compression of the subclavian artery may result in an aneurysm, which is a potential source of emboli to the hand. 


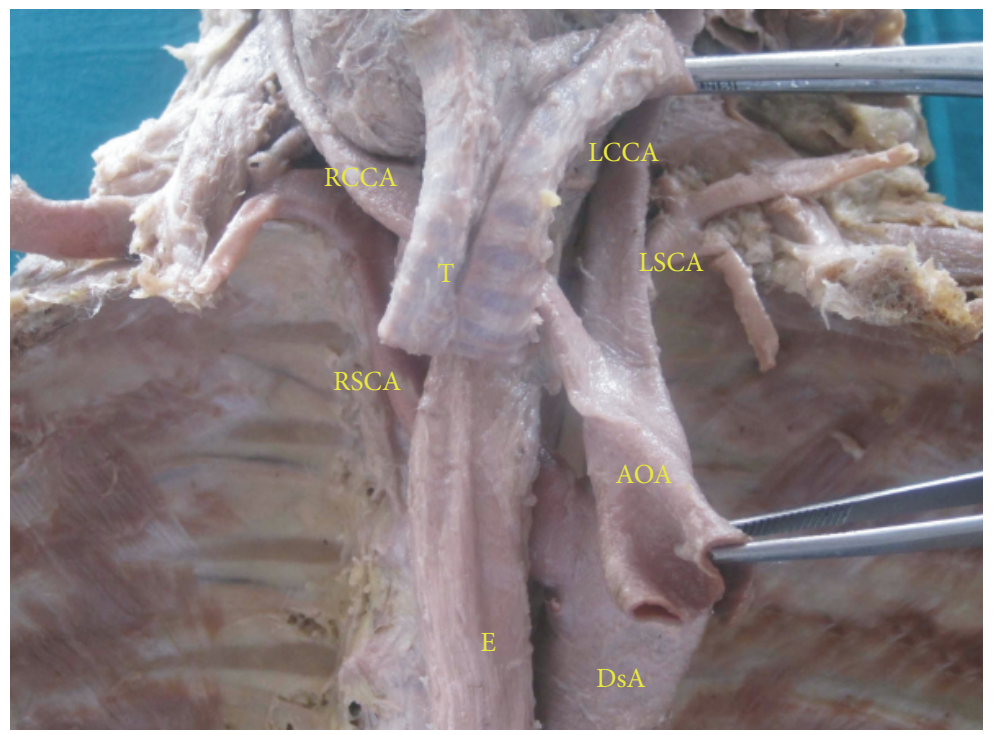

FIGURE 1: Origin of right subclavian artery from arch of aorta distal to left subclavian artery. AOA: arch of aorta, DsA: descending thoracic aorta, LSCA: left subclavian artery, LCCA: left common carotid artery, E: esophagus, T: trachea, RCCA: right common carotid artery, and RSCA: right subclavian artery.

The emboli may cause gangrene of the finger tips. In addition, repeated trauma may result in thrombosis of the subclavian artery. However critical ischemia is rare because of good collateral circulation, but the patient will complain of upper limb claudication, as well as coldness and numbness in hand [3].

3.1. Embryological Basis of Variations in the Origin of Subclavian Artery. In human embryo six pairs of aortic arches arise from the distal dilatation of the truncus arteriosus, which is known as the aortic sac. Each pair of aortic arches skirts around the foregut and connects to the dorsal aorta of the corresponding side. Normally the arch of aorta retains an arcuate character and these vessels incorporate the embryonic fourth arch of the left side. Other components of the aortic arch system disappear altogether or become radically modified [4].

The fourth aortic arch persists on both sides, but its ultimate fate is different on the right and the left sides. On the right, it forms the most proximal segment of the right subclavian artery, the distal part of which is formed by a portion of right dorsal aorta and the seventh intersegmental artery [5]. The left subclavian artery is not derived from a pharyngeal arch artery; it forms from the left seventh intersegmental artery. As the development proceeds, differential growth shifts the origin of left subclavian artery cranially. Consequently, it comes to lie close to the origin of left common carotid artery [6].

Rarely the right subclavian artery arises from distal part of aorta and passes upward and to the right behind the esophagus. Such anomalous origin takes place when the right subclavian is developed from the right seventh intersegmental artery and part of right dorsal aorta caudal to intersegmental artery; this is associated with disappearance of the right fourth arch [7] (Figure 2). This anomalous retroesophageal right subclavian artery that arises from the descending aorta rather than from the brachiocephalic artery may also compress the esophagus [4].

The origin of the retroesophageal right subclavian artery (RRSA) as the last branch of aortic arch is a common congenital aortic arch anomaly with a reported prevalence of $0.4 \%-$ $2 \%$ [8]. Of these cases $15 \%$ exhibit a retrotracheal course, with the remaining specimens resulting in the retroesophageal variant [9].

Since the incidence of a right retroesophageal subclavian artery (RRESA) is reported to range from $0.4 \%$ to $2 \%$, although described as usually asymptomatic, the RRESA can cause dysphagia lusoria as a clinical finding, due to the compression of the esophagus between the right common carotid artery and the trachea anterior to it and the right subclavian artery posterior to it [10].

The RRESA is more frequent in women and mongoloid children (Down syndrome) and is also associated with chromosome 22q11 deletion (DiGeorge syndrome) [11].

Right aortic arch with aberrant left subclavian artery and left aortic arch with aberrant right subclavian artery were seen associated with extensive airway compression in children referred for cross-sectional imaging [12]. It is reported that only $5 \%$ of these patients have associated with airway symptoms. Patients who present with airway symptoms have been found to have an associated large Kommerell's diverticulum (a pseudodiverticulum formed because of remnant of the left aortic arch at the origin of left subclavian artery) or "tight" ligamentum arteriosum [13].

The presence of a RRESA should be considered especially when ischemia of the upper limb is severe and occlusion is located at the shoulder level [14]. This is a clear example of when knowledge of an anatomical variation is helpful in clinical practice. 


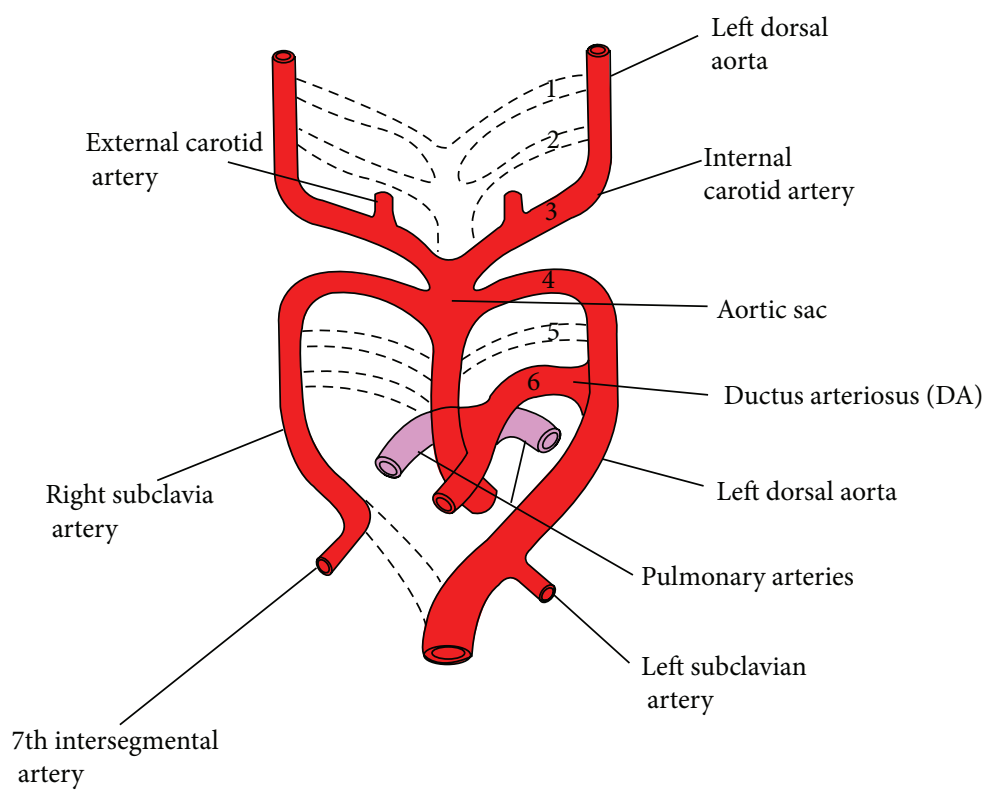

Figure 2

Correct identification of these vessels is very important for appropriate invasive techniques in order to achieve desired objectives and to avoid major complications during vascular surgery [15].

The awareness of such variations in the origin is of utmost importance during Doppler scanning of blood vessels for clinical diagnosis and surgical management.

With more and more technical advancement, clinicians dealing with the arch of aorta and the great vessels should be aware of these variations. Furthermore anatomical variations are needed to be reported constantly so that physicians and anatomists can ensure forward progress scientifically, so also knowledge of the development of the cardiovascular system could be useful for better understanding of the pathophysiology of the variation and that knowledge should be applied to recognize the variations.

\section{Conflict of Interests}

The authors declare that there is no conflict of interests regarding the publication of this paper.

\section{Acknowledgment}

The authors thank Dr. V. Arole, Professor and HOD, DYPMC, Pune, India, for her support and encouragement.

\section{References}

[1] G. J. Romanes, Cunningham's Manual of Pratical Anatomy, Oxford Medical Publications, New York, NY, USA, 15th edition, 1986.

[2] H. Gray, The Anatomical Basis of Clinical Practice, edited by S. Standring, Elsevier, Churchill Livingstone, 40th edition, 2008.

[3] L. McGregor, Synopsis of Surgical Anatomy, John Wiley \& Sons, New York, NY, USA, 12th edition, 1986.
[4] W. H. Hollinshed, Textbook of Anatomy, Lippincott-Raven, New York, NY, USA, 5th edition, 1997.

[5] T. W. Sadler, Langman's Medical Embryology, Lippincott Williams \& Wilkins, 11th edition, 2009.

[6] K. L. Moore and T. V. N. Persaud, The Developing Human: Clinically Oriented Embryology, 8th edition, 2008.

[7] A. K. Dutta, Essentials Of Human Anatomy Part 2, Current Books Internantional, Kolkata, India, 5th edition, 2009.

[8] L. Leger, "Reperage du nerf recurrent droit," La Presse Médicale, vol. 79, pp. 2383-2384, 1971.

[9] H. Lippert and R. Pabst, Arterial Variations in Man, J. F. Bregmann, Munich, Germany, 1985.

[10] N. Boas, F. Desmoucelle, V. Bernadet, and J.-C. Franceschi, "Rare cause of acute ischemia of the right upper extremity: thrombosis of a retroesophageal subclavian artery," Annals of Vascular Surgery, vol. 16, no. 3, pp. 387-390, 2002.

[11] K. Momma, R. Matsuoka, and A. Takao, "Aortic arch anomalies associated with chromosome 22q11 deletion (CATCH 22)," Pediatric Cardiology, vol. 20, no. 2, pp. 97-102, 1999.

[12] L. F. Donnelly, R. J. Fleck, P. Pacharn, M. A. Ziegler, B. L. Fricke, and R. T. Cotton, "Aberrant subclavian arteries: crosssectional imaging findings in infants and children referred for evaluation of extrinsic airway compression," American Journal of Roentgenology, vol. 178, no. 5, pp. 1269-1274, 2002.

[13] J. L. Strife, G. S. Bisset III, and P. E. Burrows, "Cardiovascular system," in Practical Pediatric Radiology, D. R. Kirks, Ed., pp. 511-613, Lippincot-Raven, Philadelphia, Pa, USA, 3rd edition, 1998.

[14] D. L. Akers Jr., R. J. Fowl, J. Plettner, and R. F. Kempczinski, "Complications of anomalous origin of the right subclavian artery: case report and review of the literature," Annals of Vascular Surgery, vol. 5, no. 4, pp. 385-388, 1991.

[15] R. Suresh, N. Ovchinnikov, and A. McRae, "Variations in the branching pattern of the aortic arch in three Trinidadians," West Indian Medical Journal, vol. 55, no. 5, pp. 351-353, 2006. 

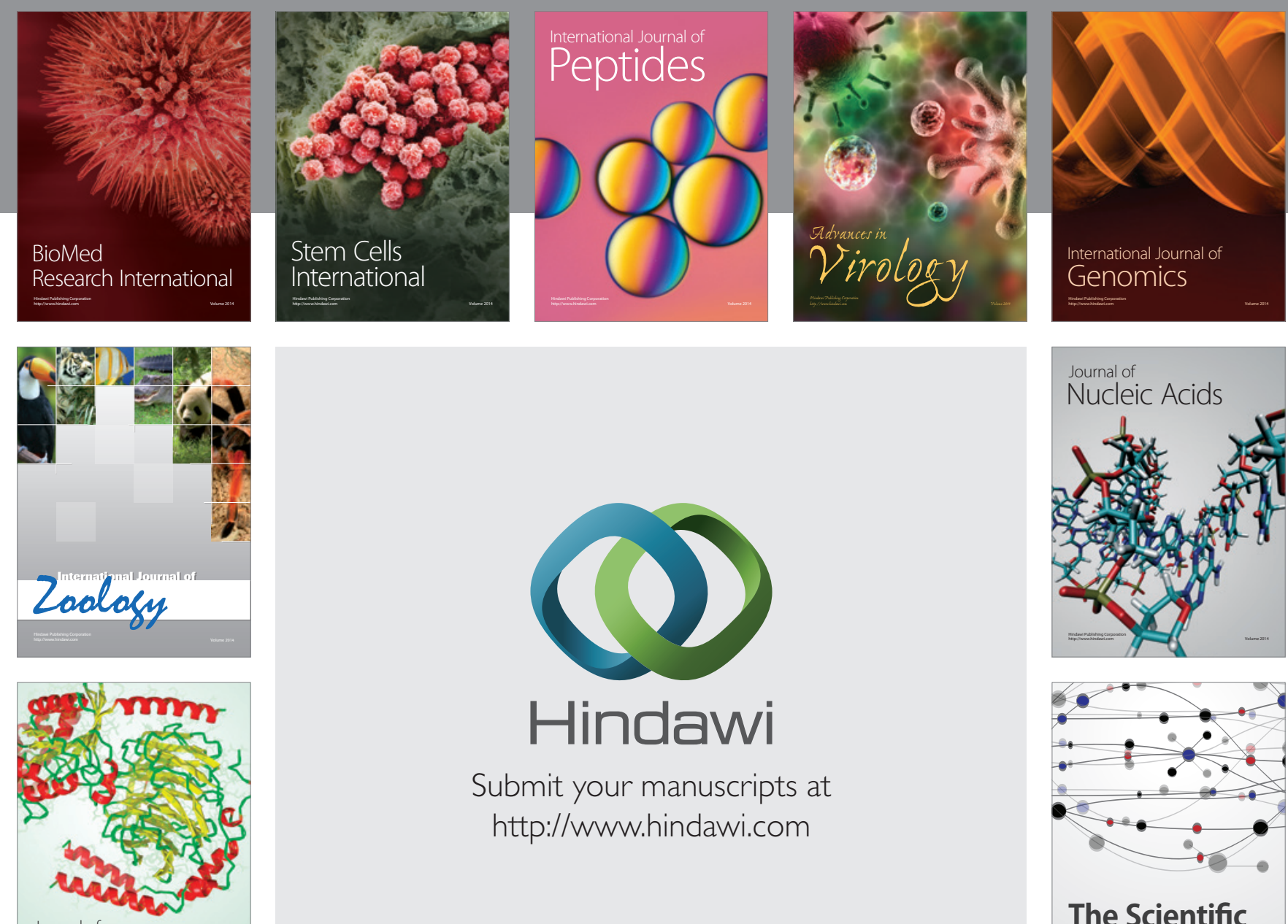

Submit your manuscripts at

http://www.hindawi.com

Journal of
Signal Transduction
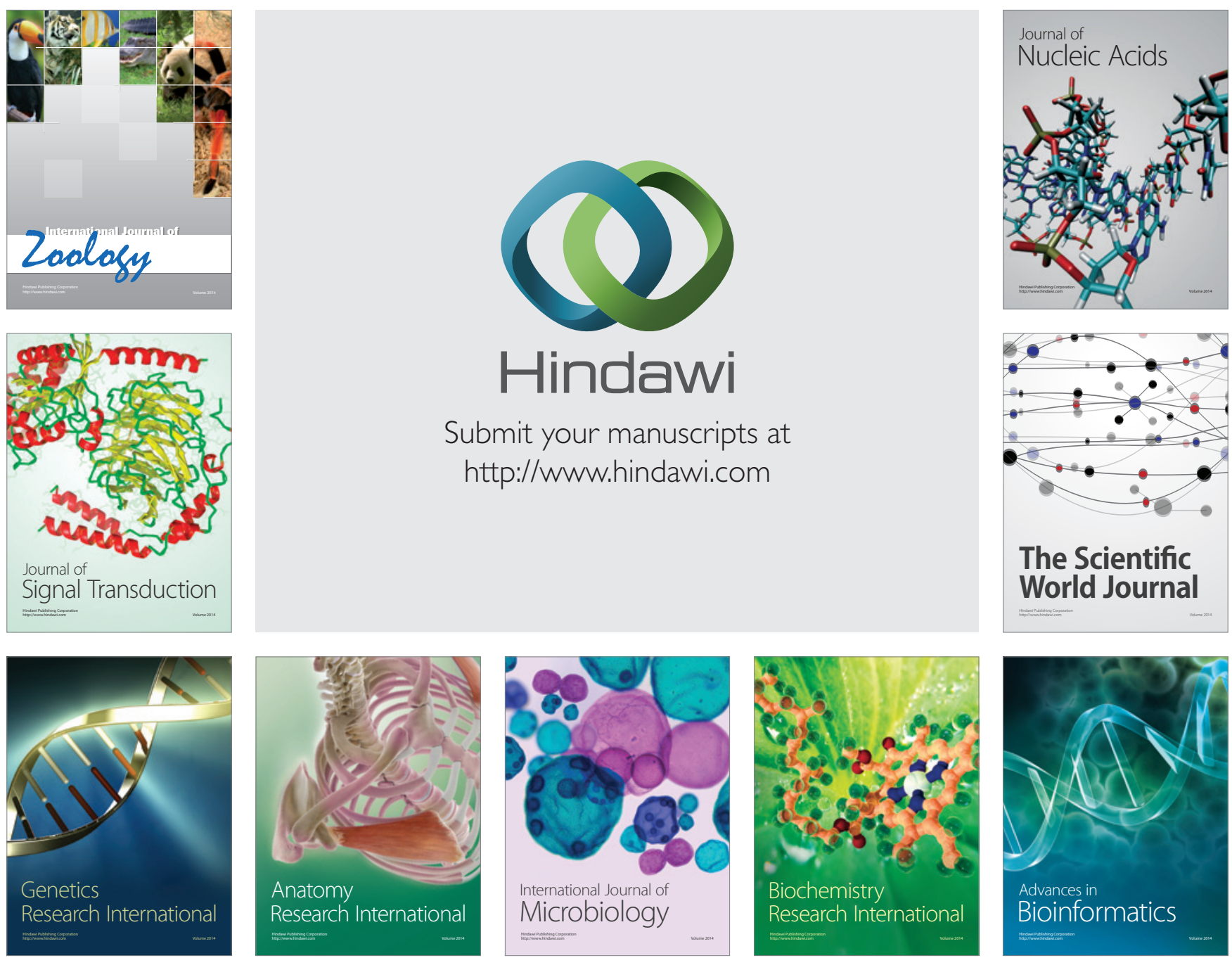

The Scientific World Journal
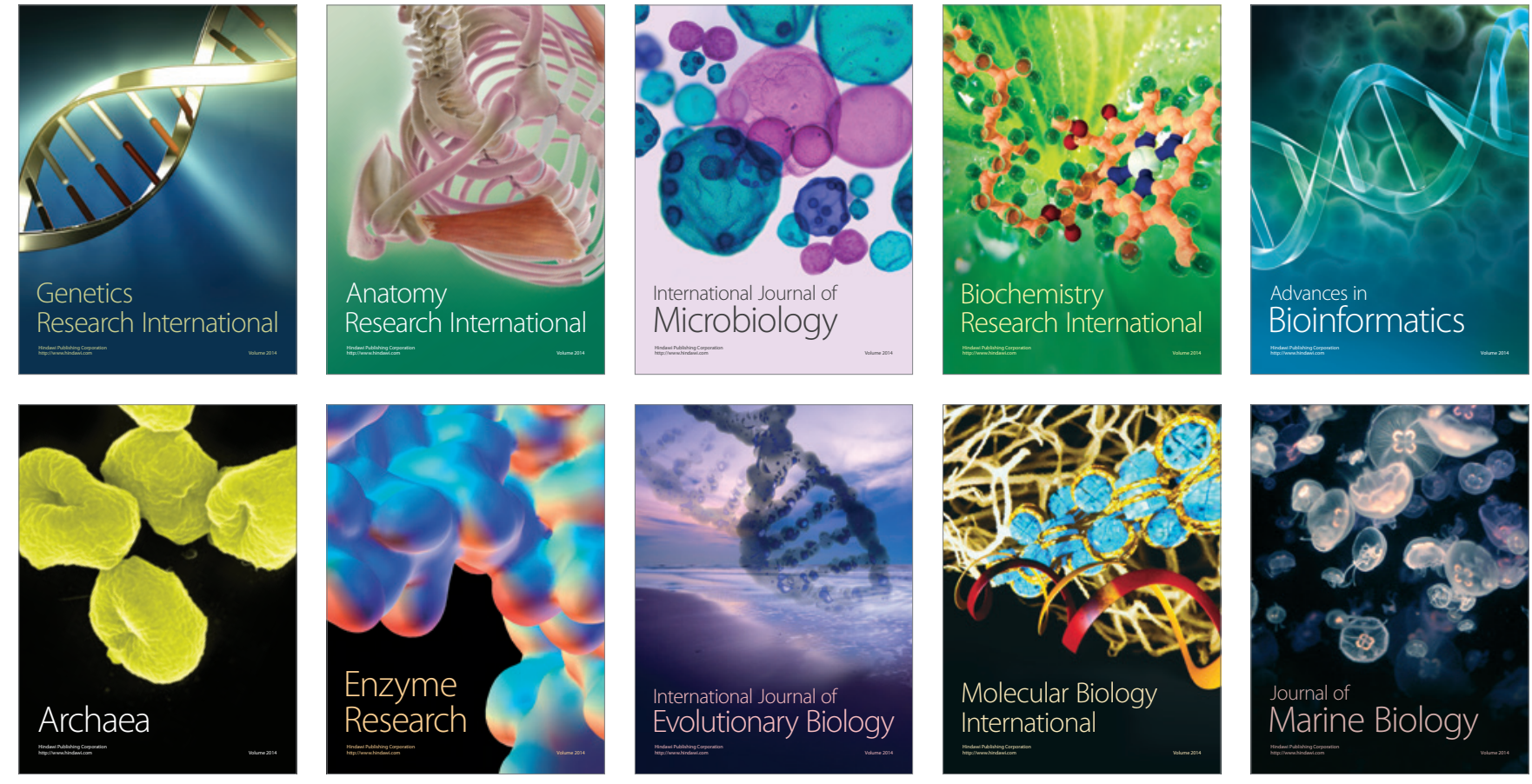